\title{
Increased mortality of acute respiratory distress syndrome was associated with high levels of plasma phenylalanine
}

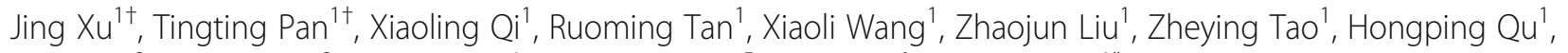
Yi Zhang ${ }^{2}$, Hong Chen ${ }^{3}$, Yihui Wang ${ }^{4}$, Jingjing Zhang ${ }^{5}$, Jie Wang ${ }^{6}$ and Jialin Liü

\begin{abstract}
Background: There is a dearth of drug therapies available for the treatment of acute respiratory distress syndrome (ARDS). Certain metabolites play a key role in ARDS and could serve as potential targets for developing therapies against this respiratory disorder. The present study was designed to determine such "functional metabolites" in ARDS using metabolomics and in vivo experiments in a mouse model.

Methods: Metabolomic profiles of blood plasma from 42 ARDS patients and 28 healthy controls were captured using Ultra-high performance liquid chromatography tandem mass spectrometry (UHPLC-MS/MS) assay. Univariate and multivariate statistical analysis were performed on metabolomic profiles from blood plasma of ARDS patients and healthy controls to screen for "functional metabolites", which were determined by variable importance in projection (VIP) scores and $P$ value. Pathway analysis of all the metabolites was performed. The mouse model of ARDS was established to investigate the role of "functional metabolites" in the lung injury and mortality caused by the respiratory disorder.
\end{abstract}

Results: The metabolomic profiles of patients with ARDS were significantly different from healthy controls, difference was also observed between metabolomic profiles of the non-survivors and the survivors among the ARDS patient pool. Levels of Phenylalanine, D-Phenylalanine and Phenylacetylglutamine were significantly increased in non-survivors compared to the survivors of ARDS. Phenylalanine metabolism was the most notably altered pathway between the non-survivors and survivors of ARDS patients. In vivo animal experiments demonstrated that high levels of Phenylalanine might be associated with the severer lung injury and increased mortality of ARDS.

Conclusion: Increased mortality of acute respiratory distress syndrome was associated with high levels of plasma Phenylalanine.

Trial registration: Chinese Clinical Trial Registry, ChiCTR1800015930. Registered 29 April 2018, http://www.chictr. org.cn/edit.aspx?.pid=25609\&htm $=4$

Keywords: Acute respiratory distress syndrome, Metabolomics, Phenylalanine, Phenylacetylglutamine, Phenylalanine metabolism, Metabolites

\footnotetext{
* Correspondence: lj111243@rjh.com.cn

${ }^{\dagger}$ Jing Xu and Tingting Pan contributed equally to this work.

1 Department of Critical Care Medicine, Ruijin Hospital, Shanghai Jiao Tong

University School of Medicine, Shanghai, China

Full list of author information is available at the end of the article
}

\section{$\triangle B M C$}

(c) The Author(s). 2020 Open Access This article is licensed under a Creative Commons Attribution 4.0 International License, which permits use, sharing, adaptation, distribution and reproduction in any medium or format, as long as you give appropriate credit to the original author(s) and the source, provide a link to the Creative Commons licence, and indicate if changes were made. The images or other third party material in this article are included in the article's Creative Commons licence, unless indicated otherwise in a credit line to the material. If material is not included in the article's Creative Commons licence and your intended use is not permitted by statutory regulation or exceeds the permitted use, you will need to obtain permission directly from the copyright holder. To view a copy of this licence, visit http://creativecommons.org/licenses/by/4.0/ The Creative Commons Public Domain Dedication waiver (http://creativecommons.org/publicdomain/zero/1.0/) applies to the data made available in this article, unless otherwise stated in a credit line to the data. 


\section{Background}

As a common cause of death in patients enrolled in intensive care units (ICUs), acute respiratory distress syndrome (ARDS) is associated with high morbidity (10.4\%) and mortality (ranging from 35 to 40\%) [1]. Unfortunately, despite many efforts made toward its diagnosis and treatment, the development of a drug treatment for ARDS remains challenging. To pursue the precise care of patients with ARDS, a better understanding of the mechanisms and accurate methods to prognosticate ARDS are required. To date, hundreds of biomarkers have been explored but very few of them have been able to successfully guide the diagnose and treatment of ARDS.

Metabolites are the downstream products of multiple intracellular biomolecules including genes and protein transporters, which enable metabolomics to serve as a remarkable tool that precisely describes "what is happening" within our body [2]. Previous studies have performed metabolomic analysis of plasma, pulmonary edema fluid and bronchoalveolar lavage fluid (BALF) in ARDS patients, preliminarily results revealed a broad range of metabolites that could help in diagnosis and stratify ARDS [3-6]. However, it is still unknown whether there exist any specific metabolites that not only help in identifying different phenotypes of ARDS but also have a crucial function in the disease process. ARDS is characterized by dysregulated immune response and diffused alveolar damage. It is well reported that some metabolites are capable of inducing inflammation and regulating the activation of immune cells $[7,8]$. Kentaro Tojo et al. have revealed that the enhancement of glycolysis attenuated the lung tissue injury by protecting alveolar epithelial cells from decline in energy [9]. Their results also indicated that metabolites played an important role in ARDS.

Therefore, our study aimed to find "functional metabolites" that could be potential therapeutic targets in ARDS. To this end, we conducted metabolomics analysis and in vivo experiments in a mouse model of ARDS. Blood plasma samples of patients with ARDS and healthy controls were collected, and metabolomics analysis was conducted to find differential metabolites and altered pathways that are associated with the ARDS mortality. Such functional metabolites were determined by selecting for the ones that were differentially expressed between ARDS patients and healthy controls and between the disease survivors and the non-survivors. Moreover, the mouse model of ARDS was established to investigate the role of identified "differential metabolites" in ARDS mortality.

\section{Methods}

\section{Study population and sample collection}

The study received approval from Ruijin Hospital Ethics Committee of Shanghai Jiao Tong University School of Medicine. All patients who met the criteria for acute respiratory distress syndrome (ARDS) according to the Berlin definition were considered for study enrollment [10]. Patients were excluded if:1) they were less than< 18 years of age; 2 ) they had any autoimmune diseases; 3 ) they were on another clinical trial; 4) they had chronic respiratory ailments; Blood samples for metabolomic analysis were collected within $48 \mathrm{~h}$ of ARDS diagnose. Volunteers from the Health Center of the Ruijin Hospital of Shanghai Jiao Tong University School of Medicine were selected as healthy controls. Altogether, a total of 42 patients and 28 healthy controls were enrolled in this study. Blood samples that were each $2 \mathrm{~mL}$ in volume were collected using heparin tube from ARDS patients and healthy controls. The samples were preserved as previously described [4]. Briefly, all samples were incubated at room temperature for $30 \mathrm{~min}$ and then centrifuged at $500 \mathrm{~g}, 4{ }^{\circ} \mathrm{C}$ for $10 \mathrm{~min}$, the supernatant is obtained and stored at $-80^{\circ} \mathrm{C}$ till further use. This trial was registered with the Chinese Clinical Trial Registry under the identification number, ChiCTR1800015930.

\section{Sample preparation}

The detail of methods for sample preparation referred to previous study [11]. Briefly, $100 \mu \mathrm{L}$ of sample was transferred to an Eppendorf (EP) tube and vortexed for $30 \mathrm{~s}$ with the addition of $300 \mu \mathrm{L}$ of methanol (containing $1 \mu \mathrm{g} / \mathrm{mL}$ internal standard). To precipitate proteins, the sample was sonicated for $10 \mathrm{~min}$ in ice-water bath and incubated for $1 \mathrm{~h}$ at $-20^{\circ} \mathrm{C}$. The sample was then centrifuged at $12000 \mathrm{rpm}$ for $15 \mathrm{~min}$ at $4{ }^{\circ} \mathrm{C}$. The resulting supernatant was transferred to a fresh glass vial for analysis. The quality control (QC) sample was prepared by mixing an equal volume of aliquot from the supernatant of each sample. For targeted UHPLC-MS/MS, a $50 \mu \mathrm{L}$ aliquot of BALF sample was transferred to an Eppendorf tube. Two hundred $\mu \mathrm{L}$ of extraction solution (acetonitrile-methanol, 1:1, and containing isotopically-labelled internal standard mixture) was added and then the samples were vortexed for $30 \mathrm{~s}$, and sonicated for $5 \mathrm{~min}$ in ice-water bath. The samples were incubated at $-40^{\circ} \mathrm{C}$ for 1 hour and centrifuged at $12000 \mathrm{rpm}$ at $4{ }^{\circ} \mathrm{C}$ for 15 min. An $80 \mu \mathrm{L}$ aliquot of the supernatant was transferred to an auto-sampler vial for UHPLC-MS/MS analysis.

\section{Untargeted metabolomics detection by UHPLC-MS/MS}

Ultra-high Pressure Liquid Chromatography-Mass Spectrum/Mass Spectrum (UHPLC-MS/MS) analysis was performed using an UHPLC system (1290, Agilent Technologies) with a UPLC HSS T3 column $(2.1 \mathrm{~mm} \times$ $100 \mathrm{~mm}, 1.8 \mu \mathrm{m})$ coupled to Q Exactive mass spectrometer (Orbitrap MS, Thermo). The mobile phase A was consist of positive $(0.1 \%$ formic acid in water)and negative modes $(5 \mathrm{mmol} / \mathrm{L}$ ammonium acetate in water). The mobile phase B was acetonitrile. The LC method used for 
detailed metabolite profiling with higher resolution was as follows: column temperature $35^{\circ} \mathrm{C}$, flow rate $0.5 \mathrm{~mL} / \mathrm{min}$, injected volume $3 \mu \mathrm{L}$. The parameters for elution gradient was as follows: $0 \sim 1.0 \mathrm{~min}, 1 \% \mathrm{~B} ; 1.0 \sim 8.0 \mathrm{~min}, 1 \% \sim 99 \%$ B; $8.0 \sim 10.0 \mathrm{~min}, 99 \% \mathrm{~B} ; 10.0 \sim 10.1 \mathrm{~min}, 99 \% \sim 1 \% \mathrm{~B}$; $10.1 \sim 12 \mathrm{~min}, 1 \% \mathrm{~B}$. The QE mass spectrometer (Orbitrap MS, Thermo) was used to acquire MS/MS spectra data under the control of the acquisition software (Xcalibur 4.0.27, Thermo). In information-dependent acquisition (IDA) mode, the acquisition software continuously evaluated the full scan MS spectrum. The ESI source conditions were set as following: sheath gas flow rate as 45 Arb, Aux gas flow rate as $15 \mathrm{Arb}$, capillary temperature $400{ }^{\circ} \mathrm{C}$, full MS resolution as 70,000, MS/MS resolution as 17,500, collision energy as $20 / 40 / 60 \mathrm{eV}$ in NCE mode, spray Voltage as $4.0 \mathrm{kV}$ (positive) or $-3.6 \mathrm{kV}$ (negative), respectively.

\section{Targeted amino acids detection by UHPLC-MS/MS}

To prepare the Standard Solution Preparation, stock solutions were prepared by dissolving or diluting each standard substance to a final concentration of $10 \mathrm{mmol} /$ L. An aliquot of the stock solutions was transferred to a $10 \mathrm{~mL}$ flask to form a mixed working standard solution. A series of calibration standard solutions were then prepared by stepwise dilution of this mixed standard solution (containing isotopically-labelled internal standard mixture in identical concentrations with the samples). The UHPLC system used was the same as described above, equipped with a Waters ACQUITY UPLC BEH Amide column. An Agilent 6460 triple quadrupole mass spectrometer (Agilent Technologies), equipped with an AJS electrospray ionization (AJS-ESI) interface, was applied for assay development. The MRM parameters for each of the targeted analytes were optimized using flow injection analysis. Several most sensitive transitions were used in the MRM scan mode to optimize the collision energy for each Q1/Q3 pair. Among the optimized MRM transitions per analyte, the Q1/Q3 pairs that showed the highest sensitivity and selectivity were selected as 'quantifier' for quantitative monitoring. The additional transitions acted as 'qualifier' for the purpose of verifying the identity of the target analytes. Agilent Mass Hunter Work Station Software (B.08.00, Agilent Technologies) was employed for MRM data acquisition and processing.

\section{Data analysis}

The raw data were converted to the mzXML format using ProteoWizard and processed by the XCMS-based R-script for peak detection, extraction, alignment, and integration. Then the Kyoto Encyclopedia of Genes and Genomes (KEGG, http://www.genome.jp/kegg/) and Human Metabolome Database (HMDB, http://www.hmdb.ca/) were applied in metabolite annotation.

\section{Mouse model and survival curve}

C57BL/6 mice (8-10 weeks old) were intratracheally injected with Pseudomonas aeruginosa $\left[2 \times 10^{6}\right.$ colonyforming units (CFU) of PAO1 strain, ATCC, Manassas, VA, USA] in $50 \mu \mathrm{L}$ phosphate-buffered saline (PBS) or just equal volume of PBS as a control. To determine the role of Phenylalanine in the mortality of ARDS, mice were pretreated with Phenylalanine (Sangon Biotech, Shanghai, China; A610422-0100) or PBS $(10 \mathrm{mg} / \mathrm{ml}$ in a total volume of $100 \mathrm{ul}$ by intravenous route) $24 \mathrm{~h}$ before the intratracheal injection of PAO1, mortality was monitored for 7 days and every $24 \mathrm{~h}$ during the week the mice were administrated with another dose of Phenylalanine or PBS until death.

\section{Assessment of lung injury}

The mice were pretreated with Phenylalanine $12 \mathrm{~h}$ before the intratracheal injection of PAO1. Every $12 \mathrm{~h}$ the mice were given another dose of Phenylalanine and then sacrificed 24h later after the infection. BALF and lung tissue were obtained to determine the lung injury. The lungs were perfused with $1.5 \mathrm{~mL}$ of PBS (3 times, 0.5 $\mathrm{mL} /$ perfusion) using a 20-gauge endotracheal catheter, followed by the collection of BALF from the right lung (the left lung was ligated with string). The supernatant of BALF samples was used to assess the protein concentration by bovine serum albumin protein assay (SigmaAldrich, St. Louis, MO, USA) and the red blood cell in pellet was removed by lysis buffer (ACK Lysis Buffer, Gibco, Grand Island, NY, USA) and then assayed for white cell counts with a cell counter (Jimbio, Jimbio Technology, Jiangsu, China). The left lung of the mice was processed for hematoxylin and eosin (HE) staining.

\section{Statistical analysis}

Univariate Analysis and multivariate statistical analysis performed by Metabo Analyst (v 4.0) were used to discriminate significant metabolites between different groups. All data were normalized to sum and pareto scaled prior to further analysis. Principle Component Analysis (PCA) was applied to find the distribution features of the dataset. Partial Least Square-Discriminant Analysis (PLS-DA) was used to determine the variable importance in projection (VIP) of each compound, the models were validated by permutation test $(n=2000)$ to avoid over-fitting. Cross validation was to determine the optimal number of components needed to build the PLS-DA mode. Only compounds with a $p$ value $<0.05$ (student's T test) and VIP value $>1.0$ were considered significantly different between groups. The Pathway analysis module was performed based on KEGG database to identify the utmost affected pathway. Receiver operating characteristic (ROC) curve and area under the ROC curve (AUROC) performed by Graphpad prism (Version 
8.0) were used to evaluate the prognostic value of potential biomarkers in patients with ARDS. The combined model of biomarkers was created by binary logistic regression analysis. The independent samples t-test and Mann-Whitney U-test were performed by SPSS 19.0 to compare normally or non-normally distributed data respectively. Categorical data were compared using the chi-square or Fisher's exact test. Kaplan-Meier plots and the log-rank test were used to compare survival between the groups treated with PBS or Phenylalanine. All tests were two-tailed, $P<0.05$ was considered to indicate statistical significance.

\section{Results}

\section{Patient characteristics}

Between May 2018 and June 2019, a total of 42 patients who fulfilled the Berlin definition of ARDS and 28 healthy volunteers were included in this study. The patients of ARDS were divided into survivors and nonsurvivors based on intensive care unit (ICU) mortality. Table 1 shows characteristics of each trial subject at the time of plasma collection including age, gender, risk factors of ARDS, severity scores of disease, laboratory results and outcomes. Appropriate measures were taken to have no difference of age and gender between the ARDS patients and the healthy controls. Compared to the survivors, the non-survivors of ARDS patients had a higher Acute Physiology and Chronic Health Evaluation II (APACHEII) score, other than that, no significant differences were found between the two groups.

\section{Multivariate data analysis of the metabolites}

We identified 414 metabolites from the plasma samples using Ultra-high performance liquid chromatography tandem mass spectrometry (UHPLC-MS/MS) assay. See Supplementary Table S1 for the abundance and distribution of these metabolites in plasma samples from patients and healthy volunteers. The differential metabolomic profiles between the ARDS patients and the healthy controls, and between the survivors and the non-survivors from the patient pool were obtained via Principal Component Analysis (PCA) and Partial Least Square-Discriminant Analysis (PLS-DA). These score plots displayed a significant separation between ARDS patients and healthy controls (Fig. 1a). Cross validation showed that five components were optimal to build the model, based on which we calculated the related statistics (Fig. 1c). The $\mathrm{R}^{2}$ was $(0.96)$ for the validity of PLS-DA model against over-fitting and the predictive ability was described by $\mathrm{Q}^{2}(0.87)$. The permutation test $(n=2000)$ with a $P$ value less than 0.05 indicated toward good predictive ability of PLS-DA models (Figure S1). Statistically significant separation of metabolomic profiles was also observed between the survivors and non-survivors of ARDS (Fig. 1b), and PLS-DA served as a valid model for discriminating the metabolites $\left(R^{2}=\right.$ 0.89, $\mathrm{Q}^{2}=0.49$ ) (Fig. 1d).

Variable Importance in the Projection (VIP) is a weighted sum of squares of the PLS loadings taking into account the amount of explained Y-variation, in each dimension (https://www.metaboanalyst.ca). The higher VIP scores of the metabolites had, the more important contribution of it in the differences between groups. A VIP plot generated from the PLS-DA models ranked individual metabolites for their power to discriminate ARDS from controls (Fig. 2a). It can be seen that phosphatidylcholine (PC) (16:0/0:0), isoleucine, D-phenylalnaine and L-Gulose in the plasma were mainly contributed to the metabolic differences between ARDS patients and healthy controls (VIP > 3.0). The differences in the metabolic profiles of the survivors and the non-survivors of ARDS patients are shown in Fig. 2b. They were mainly attributed to D-Phenylalanine, myristic acid and oleic acid in the plasma (VIP > 3.0). The heatmap shows the abundance of top 30 metabolites in all individuals based on VIP scores. We found several glycerophospholipids like 1-Linoleoylglycerophosphocholine, $\mathrm{PC}(16,0 / 0: 0)$, Phosphatidylcholine lyso18:2, and LysoPC20:4(5Z,8Z,11Z,14Z) that were significantly downregulated in the ARDS patients compared to the healthy controls (Fig. 2c). The levels of D-Phenylalanine and Phenylalanine increased in the non-survivors compared to the survivors while a group of short peptides containing different amino acid residues downregulated in the non-survivors (Fig. 2d).

\section{Screening of differentially expressed metabolites as potential mortality predictors for ARDS}

We next investigated the potential biomarkers and mortality predictors of ARDS patients. To preliminary screen the differential metabolites, we selected the metabolites that had a $P$ value less than 0.05 (calculated by Student's t-test) and a VIP score greater than 1.0 (calculated using PLS-DA model). The differential metabolites between ARDS patients and healthy controls are displayed in Table 2. Similarly, the metabolites that were found to distinguish the non-survivors from the survivors of ARDS are shown in Table 3. Among all those selected compounds, Phenylalanine, D-Phenylalanine, Phenylacetylglutamine and a short peptide (Gly Pro Gly Lys) were identified in both groups (ARDS vs healthy controls and survivors vs non-survivors) (Fig. 3a). As Phenylalanine, D-Phenylalanine and Phenylacetylglutamine are all involved in the Phenylalanine metabolism, we focused on these three metabolites. The box whisker plots showed that all of three compounds had significant higher concentration in the non-survivors compared to the survivors (Fig. 3b-d). Phenylalanine and D-Phenylalanine had higher levels in the ARDS patients compared to the healthy controls (Fig. 3b-c). We also compared these 
Table 1 Demographics of ARDS patients and healthy controls

\begin{tabular}{|c|c|c|c|c|c|}
\hline & Controls & Acute respira & ss syndrome & & \\
\hline & & $\overline{\text { Total }}$ & Survivors & $\overline{\text { Non-survivors }}$ & \\
\hline Numbers & 28 & 42 & 27 & 15 & \\
\hline Age (year) & $62.3 \pm 15.3$ & $64.2 \pm 14.0$ & $63.07 \pm 15.5$ & $66.3 \pm 10.9$ & 0.48 \\
\hline Gender, Female, n (\%) & $17(60.7 \%)$ & $28(66.7 \%)$ & $19(70.4 \%)$ & $9(69.2 \%)$ & 0.36 \\
\hline Risk factors of ARDS, $n(\%)$ & & & & & \\
\hline Pneumonia & & $27(64.3 \%)$ & $18(66.7 \%)$ & $9(60 \%)$ & 0.74 \\
\hline Aspiration & & $1(2.4 \%)$ & $0(0 \%)$ & $1(6.7 \%)$ & 0.38 \\
\hline Extrapulmonary sepsis & & $6(14.3 \%)$ & $4(14.8 \%)$ & $2(13.3 \%)$ & 0.64 \\
\hline Pancreatitis & & $48(8.9 \%)$ & $3(11.1 \%)$ & $1(6.7 \%)$ & 0.55 \\
\hline Trauma or hemorrhagic shock & & $2(4.8 \%)$ & $1(3.7 \%)$ & $1(6.7 \%)$ & 0.59 \\
\hline others & & $2(4.8 \%)$ & $1(3.7 \%)$ & $1(6.7 \%)$ & 0.59 \\
\hline The severity of ARDS & & & & & \\
\hline Mild & & $7(16.7 \%)$ & $4(14.8 \%)$ & $3(20 \%)$ & 0.69 \\
\hline Moderate & & $24(57.1 \%)$ & $19(70.4 \%)$ & $5(18.5 \%)$ & $0.02 \#$ \\
\hline Severe & & $11(26.2 \%)$ & $4(14.8 \%)$ & $7(25.9 \%)$ & 0.07 \\
\hline APACHE II score & & $17.1 \pm 6.5$ & $15.6 \pm 6.1$ & $19.7 \pm 6.7$ & $0.05^{*}$ \\
\hline $\mathrm{PaO} 2 / \mathrm{FiO}_{2}$ & & $153.5 \pm 68.3$ & $167.6 \pm 62.9$ & $128.1 \pm 72.3$ & 0.07 \\
\hline $\mathrm{CRP}, \mathrm{mg} / \mathrm{dL}$ & & $97.3 \pm 69.5$ & $100.0 \pm 63.0$ & $91.9 \pm 83.0$ & 0.73 \\
\hline $\mathrm{PCT}, \mathrm{ng} / \mathrm{mL}$ & & $46.5 \pm 84.8$ & $52.9 \pm 93.0$ & $34.9 \pm 69.4$ & 0.52 \\
\hline Leukocytes, $\times 10^{9} / \mathrm{L}$ & & $12.6 \pm 5.9$ & $13.1 \pm 5.8$ & $11.8 \pm 6.1$ & 0.50 \\
\hline Neutrophils, $\times 10^{9} / \mathrm{L}$ & & $11.1 \pm 5.4$ & $12.0 \pm 5.8$ & $9.4 \pm 4.4$ & 0.16 \\
\hline Lymphocytes, $\times 10^{9} / \mathrm{L}$ & & $2.3 \pm 2.9$ & $2.5 \pm 3.2$ & $1.9 \pm 2.1$ & 0.51 \\
\hline Albumin $(\mathrm{g} / \mathrm{L})$ & & $51.6 \pm 53.5$ & $50.8 \pm 56.9$ & $52.8 \pm 50.4$ & 0.93 \\
\hline Prealbumin (mg/L) & & $94.6 \pm 54.5$ & $100 \pm 55.1$ & $86.5 \pm 55.0$ & 0.52 \\
\hline Ventilator free days & & $7.5 \pm 13.2$ & $9.6 \pm 12.7$ & $3.7 \pm 13.7$ & 0.17 \\
\hline ICU stay, days & & $32.4 \pm 26.8$ & $25.8 \pm 30.8$ & $23.1 \pm 16.9$ & 0.33 \\
\hline $\mathrm{Vt} / \mathrm{Bwt}$ & & $7.03 \pm 1.8$ & $7.2 \pm 2.0$ & $6.9 \pm 1.5$ & 0.72 \\
\hline Inhaled nitric oxide therapy & & 0 & 0 & 0 & / \\
\hline Prone position & & $4(9.5 \%)$ & $4(14.8 \%)$ & 0 & 0.28 \\
\hline ECMO & & $2(4.8 \%)$ & $1(3.7 \%)$ & $1(6.7 \%)$ & 1.0 \\
\hline
\end{tabular}

Quantitative data are presented as mean $\pm S D$, Qualitative data are presented as number (\%), $P$-value for the survivors and non-survivors of ARDS; $A P A C H E$ acute physiologic and chronic health evaluation, $C R P$ C-reactive protein, $P C T$ procalcitonin, $E C M O$ extracorporeal Membrane Oxygenation; ${ }^{*} P<0.05$ tested by student $T$ test. \# $P<0.05$ tested by chi-square test

metabolites in ARDS patients with different severity (mild moderate and severe) according to Berlin's definition. The levels of Phenylacetylglutamine were markedly lower in moderate ARDS patients compared to severe ARDS patients (Figure S2). There was no significant difference among the concentration of Phenylalanine and D-Phenylalanine in three groups (Figure S2). To investigate the effect of these three differentially expressed metabolites in predicting the mortality of ARDS, we plotted a ROC curve (Fig. 3e) to assess the sensitivity and specificity. The area under the ROC curve (AUC) of Phenylalanine, D-Phenylalanine and Phenylacetylglutamine is $0.803,0.785$ and 0.709 , respectively, indicating that
Phenylalanine had the highest efficacy in predicting the mortality of ARDS. In the combined model the AUC was 0.882 .

Pathway analysis reveals the phenylalanine pathway to be one of the most significantly altered pathways in ARDS patients

To gain the functional interpretation of these numerous compounds in ARDS, we applied Pathway Analysis of the metabolites identified in our data. The pathways that altered between ARDS and healthy controls or between survivors and non-survivors were listed in Table S2. Figure 4a and $\mathrm{b}$ display all matched pathways. We screened the 

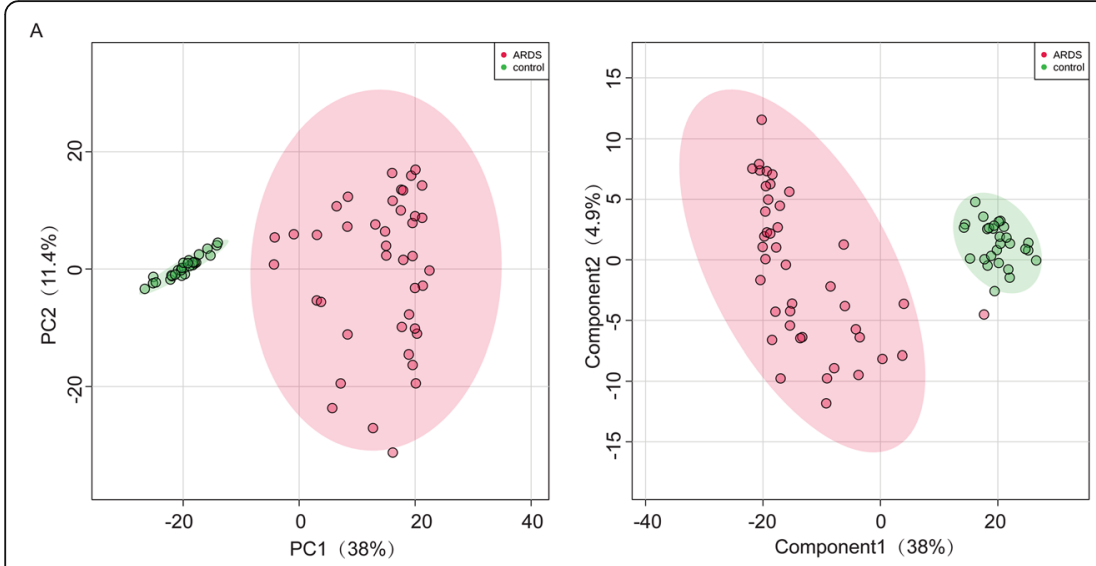

C
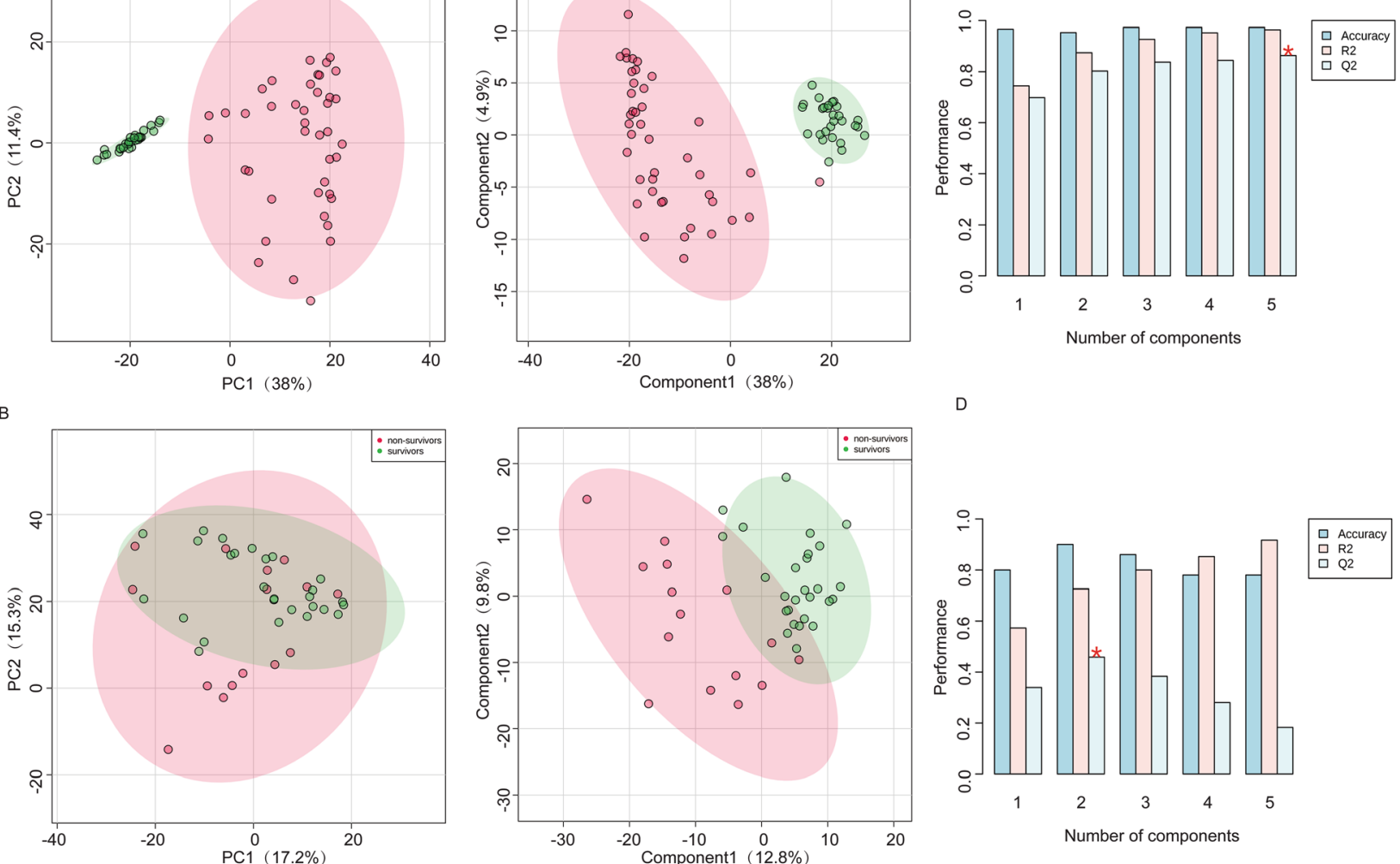

D

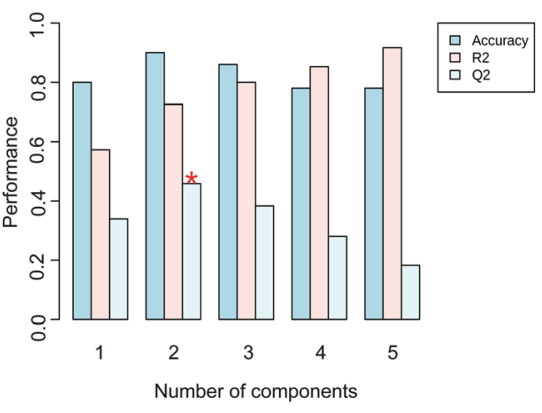

Fig. 1 Multivariate data analysis of the metabolites. a Principal Component Analysis (PCA) scores plots (left panels) and Partial Least Square-Discriminant Analysis (PLS-DA) scores plots (right panels) of ARDS patients vs healthy controls. Shaded areas are the $95 \%$ confidence regions of each group. b PCA and PLS-DA scores plots of the survivors vs the non-survivors of ARDS. Shaded areas are the $95 \%$ confidence regions of each group. c The cross validation test for PLS-DA model of the ARDS patients vs the healthy controls. $\mathbf{d}$ The cross validation test for PLS-DA model of the survivors vs non-survivors of ARDS. Deep blue represents the cross-validated $R^{2}$ (also known as $Q^{2}$ ),pink represent the sum of squares captured by the model $\left(R^{2}\right)$, and light blue represents the prediction accuracy. Red star indicates the best component number in building the model. In PLS-DA of the ARDS patients vs the healthy controls analysis, $R^{2}=0.93$ and $Q^{2}=0.86$, the survivors vs the non-survivors of $A R D S$ analysis, $R^{2}=0.89$ and $Q^{2}=0.49$, respectively

pathways with an impact factor greater than 0 and a $P$ value less than 0.05 , the top 5 pathways in the group of ARDS versus healthy controls were 1 ) purine metabolism pathway, 2) Phenylalanine, tyrosine and tryptophan biosynthesis pathway, 3) histidine metabolism pathway, 4) Phenylalanine metabolism pathway and 5) glycerophospholipid metabolism pathway. In the survivors versus non-survivors group, only three pathways satisfied the standards of screening, they were 1) D-Glutamine and D-glutamate metabolism pathway, 2) Phenylalanine, tyrosine and tryptophan biosynthesis pathway, and 3) Phenylalanine metabolism pathway. Figure 4c shows that in both the ARDS versus healthy controls group and the non-survivors versus survivors group, Phenylalanine metabolism pathway, Phenylalanine, tyrosine and tryptophan biosynthesis pathway were identified as the most significantly altered pathways. To visualize the change of metabolites in the most significantly altered pathways between non-survivors and survivors, the metabolic network was plotted (Fig. 4d), all the matched metabolites in our data that involved in the pathways were marked in red, yellow and green according to the different levels of significance. In Phenylalanine metabolism pathway, five compounds were matched and three of them (Phenylalanine, D-Phenylalanine and phenylacetylglutamine) upregulated while tyrosine and hippurate were downregulated in the non-survivors compared to the survivors. In Phenylalanine, tyrosine and tryptophan biosynthesis pathway, three metabolites were matched with our data, L-Tryptophan and Phenylalanine levels increased while Tyrosine levels decreased in the non-survivors. In D-glutamine and Dglutamate metabolism pathway, only one metabolites (glutamine) was matched and was found to be significantly downregulated in the non-survivors.

\section{Phenylalanine administration increased the lung injury and mortality of ARDS}

The differential metabolites and pathway analysis indicated that Phenylalanine, D-Phenylalanine and 


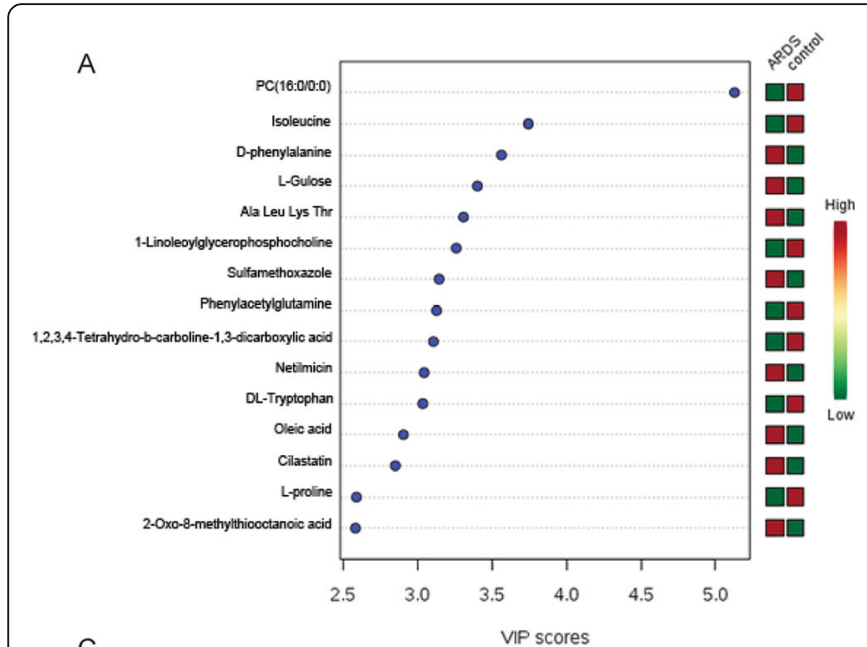

\section{B}

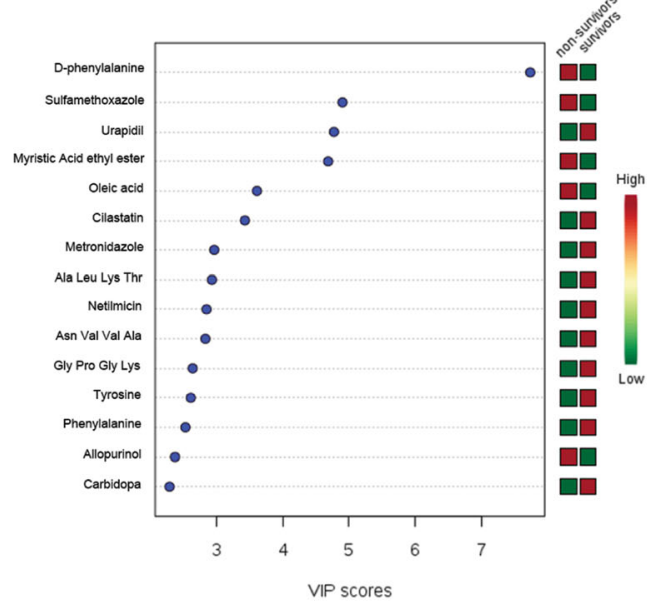

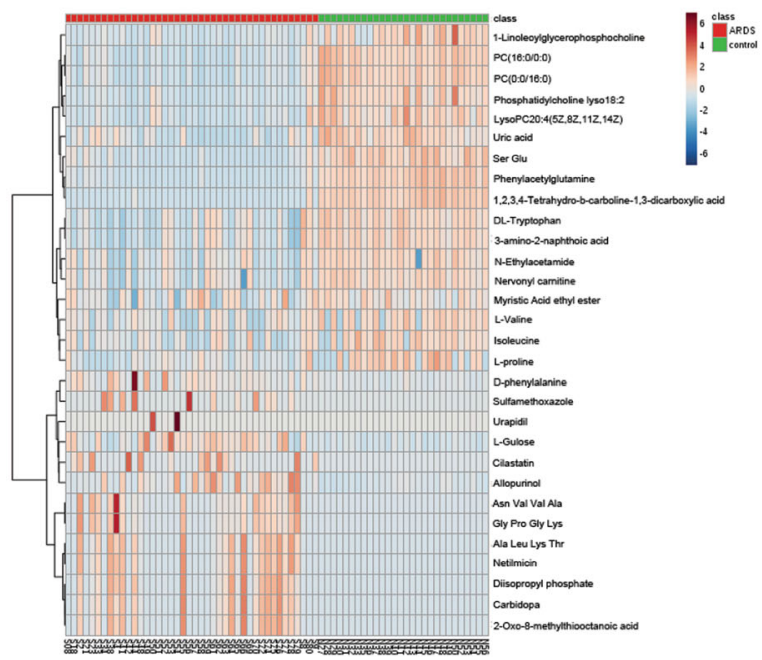

$\mathrm{D}$

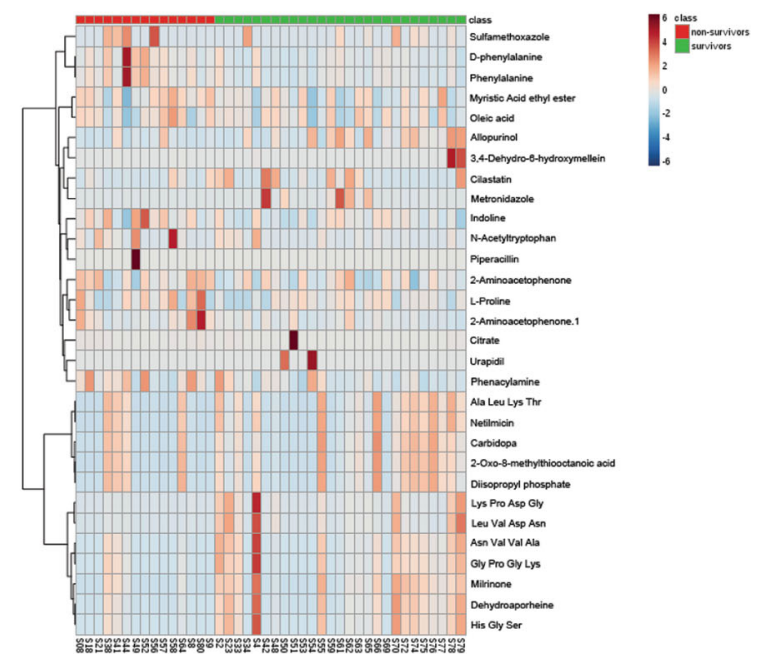

Fig. 2 VIP score plot and Heatmap of metabolites. a Variable Importance in Projection (VIP) score plot of the metabolites that differed in ARDS patients vs healthy controls. b Variable Importance in Projection (VIP) score plot of the metabolites that differed in survivors vs the non-survivors of ARDS. c The heatmap showing abundance of the top 30 metabolites based on VIP scores of ARDS patients vs healthy controls. d The heatmap showing abundance of the top 30 metabolites based on VIP scores of the survivors vs the non-survivors of ARDS

Phenylacetylglutamine might play a role in ARDS. DPhenylalanine and Phenylacetylglutamine are products of Phenylalanine, and the ROC analysis showed that Phenylalanine had the highest accuracy of predicting the mortality. Therefore, we investigated whether Phenylalanine increased the mortality of ARDS in a mouse model. The ARDS mouse model was established and Phenylalanine was administrated by intravenous injection. As shown in Fig. 5a, mice were pretreated with Phenylalanine at $0 \mathrm{~h}$ and received intratracheal injection of Pseudomonas aeruginosa (PAO1) at $24 \mathrm{~h}$. The ARDS mice and sham mice were administrated with either a dose of Phenylalanine or PBS every $24 \mathrm{~h}$, following which the mice started to die at $24 \mathrm{~h}$ after injection of PAO1. A significant increase of mortality rate was observed around $54 \mathrm{~h}$, at which time $48 \%$ of the mice injected with Phenylalanine and $30 \%$ of mice injected with PBS found dead. At 7 days after PAO1 injection, the Phenylalanine group had significantly lower survival rate (8\%) compared to those treated with PBS (14\%) (Fig. 5b). The same amount of Phenylalanine was administrated in sham mice without ARDS every $24 \mathrm{~h}$ for 7 days and no death was observed during the period (Fig. $5 \mathrm{~b})$, confirming that the concentration of Phenylalanine we used was not lethal in control mice. To determine the changes of Phenylalanine and other related amid acids in BALF after the Phenylalanine injection, we performed targeted UHPLC-MS/MS to assay Phenylalanine, glutamine, tyrosine and tryptophan in sham mice after two doses of intravenous injection of Phenylalanine. The Phenylalanine in BALF significantly increased after injection, whereas the tyrosine (downstream products of Phenylalanine) did not change, indicating that intravenously injection of Phenylalanine increased the levels of 
Table $\mathbf{2}$ List of the differentiated metabolites between ARDS patients and healthy controls

\begin{tabular}{lll}
\hline Name & VIP value & $P$ value \\
\hline Oleic acid & 4.0374 & $2.64 \mathrm{E}-06$ \\
D-Phenylalanine & 3.4261 & $2.88 \mathrm{E}-06$ \\
L-Gulose & 2.4759 & $3.78 \mathrm{E}-10$ \\
PC(16:0/0:0) & 2.162 & $1.11 \mathrm{E}-12$ \\
Tyrosine & 1.8285 & $1.73 \mathrm{E}-05$ \\
1,2,3,4-Tetrahydro-beta-carboline-1, & 1.6314 & $9.87 \mathrm{E}-40$ \\
3-dicarboxylic acid & & \\
Palmitoleic acid & 1.6274 & $3.41 \mathrm{E}-05$ \\
Phenylacetylglutamine & 1.5472 & $1.25 \mathrm{E}-33$ \\
1-Linoleoylglycerophosphocholine & 1.2548 & $4.13 \mathrm{E}-06$ \\
L-Carnitine & 1.1955 & $1.06 \mathrm{E}-04$ \\
Creatine & 1.1823 & $9.14 \mathrm{E}-04$ \\
Tagatose & 1.0799 & $4.05 \mathrm{E}-05$ \\
Phenylalanine & 1.0284 & $2.20 \mathrm{E}-06$ \\
Ala Leu Lys Thr & 2.0248 & $2.41 \mathrm{E}-05$ \\
Asn Val Val Ala & 1.2398 & 0.000491 \\
\hline The exotic drugs were not shown in this table & &
\end{tabular}

this amino acid in lung. We found the levels of glutamine and tryptophan (not significantly) in BALF also increased after injection of Phenylalanine (Figure S3). The lung injury of mice were assayed $24 \mathrm{~h}$ post injection with PAO1, protein and white cell counts in bronchoalveolar lavage fluid (Fig. 5b-c) increased in ARDS mice injected with Phenylalanine compared to those treated with PBS, indicating that Phenylalanine increased the recruitment of inflammatory cells and impairment of alveolar epithelial cells. The pathological changes in lungs of mice with ARDS assayed by hematoxylin-eosin (HE) staining

Table 3 List of the differentiated metabolites between survivors and non-survivors of ARDS patients

\begin{tabular}{lll}
\hline Name & VIP value & $P$ value \\
\hline D-Phenylalanine & 6.0449 & 0.01706 \\
Phenylacetylglutamine & 3.3932 & 0.001569 \\
Phenylalanine & 1.8439 & 0.011172 \\
Dehydroaporheine & 1.8075 & 0.005813 \\
Choline & 1.1339 & 0.020561 \\
2-Aminoacetophenone & 1.1019 & 0.016276 \\
Val Cys Thr & 1.1417 & 0.0085869 \\
Trp Trp Leu & 1.2142 & 0.034223 \\
Lys Pro Asp Gly & 1.8047 & 0.038664 \\
Leu Val Asp Asn & 1.5179 & 0.025572 \\
Gly Pro Gly Lys & 2.2304 & 0.026714 \\
Asn Val Val Ala & 2.4713 & 0.017745 \\
\hline
\end{tabular}

The exotic drugs were not shown in this table indicated severer destruction of alveoli and inflammation in Phenylalanine group than that in PBS group (Fig. 5e). The lung injury in sham mice treated with Phenylalanine and PBS had no significant differences. Together, these results confirmed that Phenylalanine administration increased the lung injury and mortality of ARDS mice but not in sham mice.

\section{Discussion}

In this study, we found that the global metabolomic profile of blood plasma from an ARDS patient was remarkably different than that of a healthy volunteer. Striking difference in the metabolomic profile was also noticed between the survivors and the non-survivors of this disease. By metabolomics analysis, we uncovered metabolites differences as well as the altered pathways that attributed to the mortality of ARDS patients. Of note, we showed that the level of Phenylalanine increased in the non-survivors compared to the survivors and we confirmed using a mouse model that Phenylalanine administration increased the lung injury and mortality of mice with ARDS.

Phenylalanine is an essential amino acid required for biosynthesis of neurotransmitters [12]. The high serum Phenylalanine is neurotoxic, producing intellectual disability, and other neurologic features [13]. Recently, the role of Phenylalanine in acute inflammatory diseases has been increasingly investigated. It was found that the serum Phenylalanine increased in patients post trauma or with sepsis [14] and was associated with the activation of immune response $[15,16]$. In a recent study, ShieShian Huang and et al. uncovered that high levels of serum Phenylalanine was associated with high mortality risk in patients with severe infection [17]. Consistent with our findings of Phenylalanine, Akhila Viswan and his colleagues reported that the relative concentration of Phenylalanine increased in non-survivors of ARDS patients compared to survivors [4]. Another study also revealed the upregulation of Phenylalanine metabolism in patients with more severe ARDS compared to those with less severity. Although these studies provided supportive evidence of the associations between Phenylalanine and the prognosis of ARDS, it is hard to determine whether the increased Phenylalanine was just a phenotype reflecting the severity or it also played a role in the process of ARDS [3]. Through the injection of Phenylalanine in ARDS mice, our study demonstrated that Phenylalanine played a detrimental role of in ARDS.

The accumulation of Phenylalanine could be a consequence of reduced activity of Phenylalanine 4hydroxylase (PHA) and its cofactor 5,6,7,8-tetrahydrobiopterin (BH4) [18]. These enzymes convert Phenylalanine into tyrosine. To be noted that, in our results, the levels of tyrosine decreased in the non-survivors 


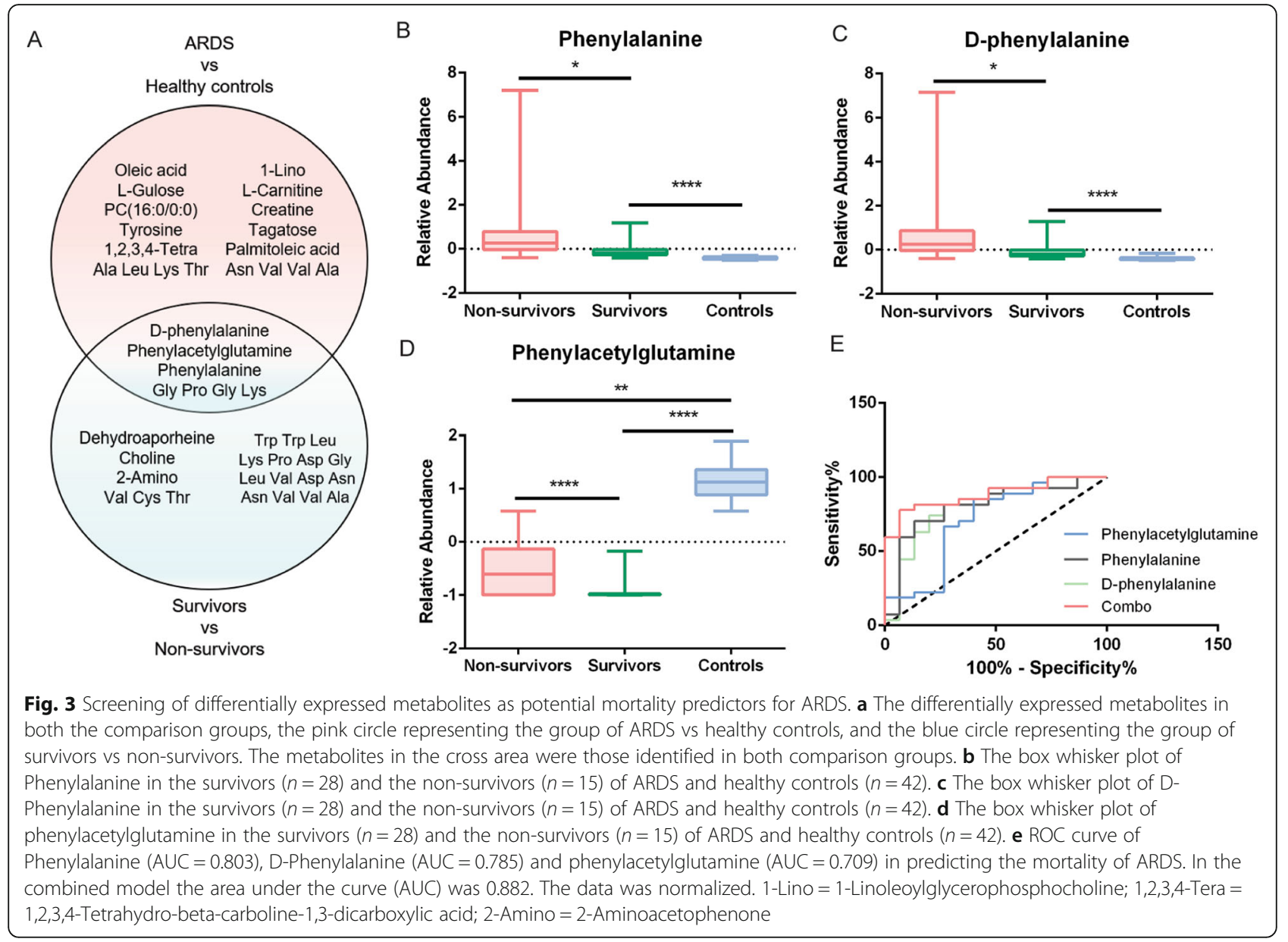

compared to the survivors of ARDS patients (not a significant difference though), which means the turnover of Phenylalanine into tyrosine in the non-survivors may be impaired. A number of studies have indicated that the deficiency of PHA and BH4 in inflammatory disease may be due to the overwhelming production of reactive oxygen species (ROS) $[12,19]$. Therefore, it was possible but not conclusive that the increased Phenylalanine in the non-survivors of ARDS was due to the deficiency of PHA and BH4 induced by inflammation.

The accumulated Phenylalanine in turn amplified the already existing inflammation. A study found that intraperitoneal injection of Phenylalanine increased IL-2 secretion in early pregnancy mice, which implied that Phenylalanine could sustain $\mathrm{T}$ cell proliferation and thereby enhance the adaptive immune in response [20]. More recently, Ming Jiang et.al demonstrated that Phenylalanine enhanced the innate immune response of the host [21]. Multiple immunological processes involving neutrophils, macrophages, and dendritic cells participate in mediating lung tissue injury in ARDS [22]. Thus the activation of immune response by Phenylalanine may exert negative impact on ARDS. Our data showed that Phenylalanine increased the lung injury of mice with ARDS, which we speculated was associated with the role of Phenylalanine in promoting inflammation.

Phenylacetylglutamine was recently reported to be a gut microbiota generated metabolite fermented from dietary Phenylalanine [23]. The levels of phenylacetylglutamine significantly decreased in human after a 7-day course of oral broad spectrum antibiotics cocktail. Further investigation revealed microbial porA gene in Clostridium facilitated the conversion of Phenylalanine into phenylacetylglutamine [23]. In our study, the levels of phenylacetylglutamine were lower in ARDS than that in controls, which was presumably due to the damage of gut microbiota caused by antibiotics use in ARDS patients. Phenylacetylglutamine was demonstrated to enhance platelet activation-related phenotypes and thrombosis potential in animal models of arterial injury [23]. Pulmonary thrombosis is common in sepsis-induced ARDS shown by human and animal studies [24]. Platelets could promote pulmonary vascular damage in sepsis induced ARDS, thereby aggravating the lung injury [25]. We found the non-survivors of ARDS had higher levels of phenylacetylglutamine than survivors, the mechanisms underlying may be related to the roles of phenylacetylglutamine in modulating platelets and thrombosis. 


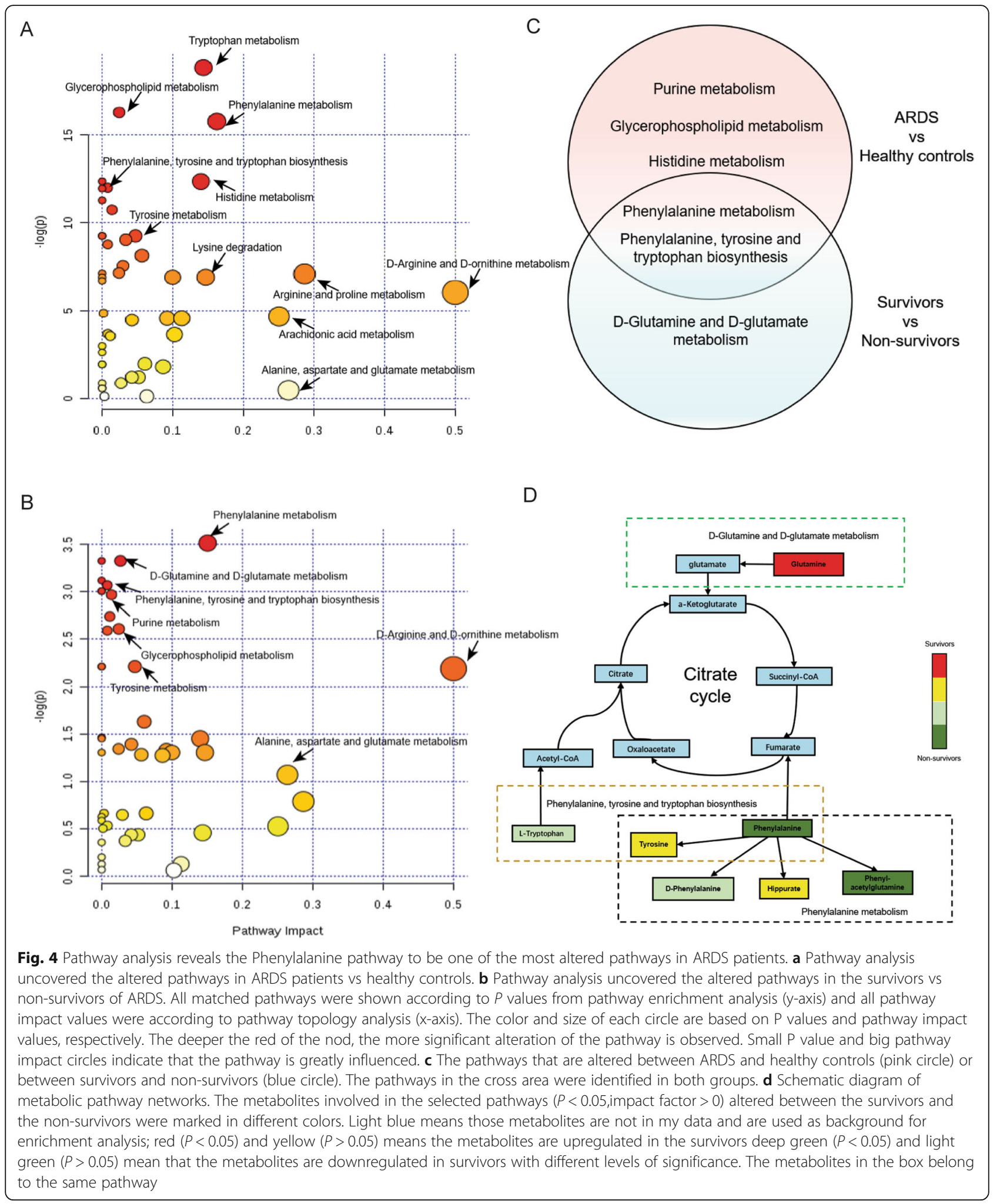

In the last decades, a broad range of drug therapies emerged for improving ARDS, but none showed efficacy in phase II and III trials [26-31]. Given the high mortality rate of ARDS, even a small improvement can save many lives. Nutritional input may be a good choice as a new adjuvant strategy for ARDS, since most metabolites can be manipulated by simply controlling the uptake from food. Omega-3 fatty acids were once used for 
A
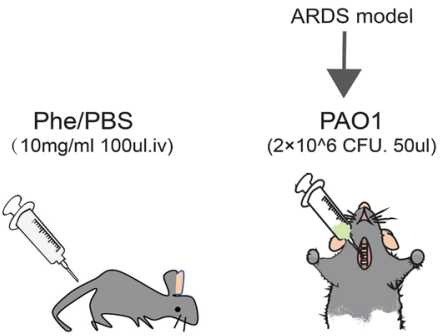

Phe/PBS $\times 2$

Phe/PBS $\times \mathrm{N}$

(10mg/ml 100uliv) (10

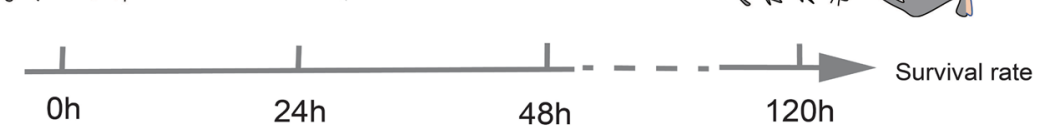

B

C
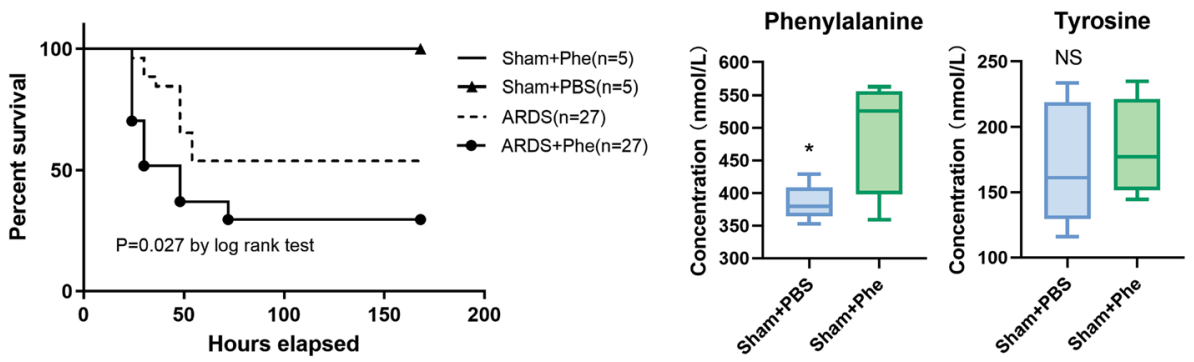

D

$\mathrm{F}$
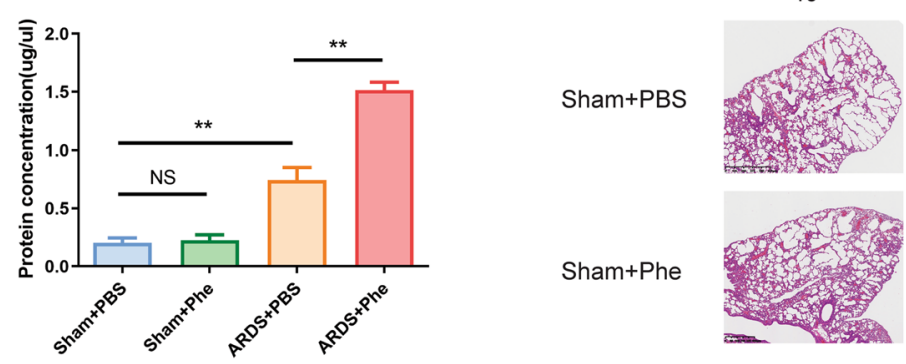

$100 \times$

E

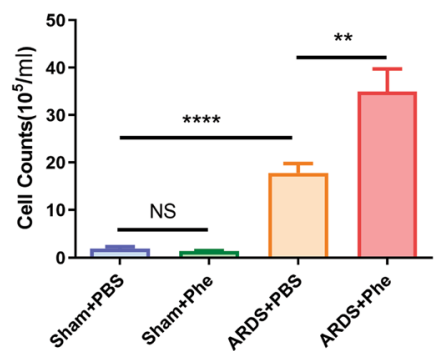

ARDS+PBS
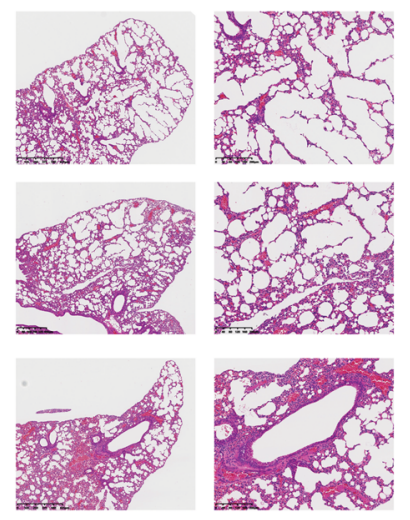

ARDS+Phe
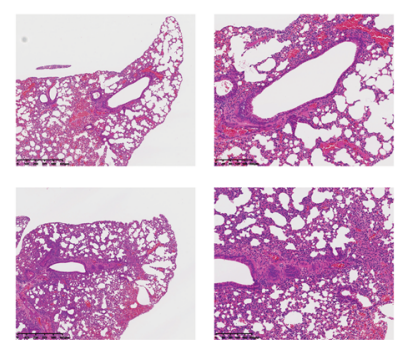

Fig. 5 Phenylalanine administration increased the lung injury and mortality of ARDS. a The protocol of Phenylalanine administration and ARDS model establishment $\mathbf{b}$ The survival rate of ARDS mice ( $n=27 /$ group) and Sham mice $(n=5 /$ group) treated with Phenylalanine or PBS. c The levels of Phenylalanine and tyrosine in BALF of sham mice treated with Phenylalanine or PBS $(n=4-5 / g r o u p)$. d The protein concentration in bronchoalveolar lavage fluid (BALF) ( $n=3-5$ /group). e The white cell counts in BALF ( $n=3-5 / g r o u p)$. f The hematoxylin and eosin staining of lung tissue ( $n=3-5$ /group) Phe = Phenylalanine. Each value represents the mean \pm SEM of one of the three independent experiments. Kaplan Meier Survival analysis and comparisons were performed by log-rank test

treatment of ARDS but ended up with a negative results, one of the reasons for this could be the failure of identifying specific metabolites in ARDS patients [32]. However, with the development of metabolomics, we are able to screen the most specific metabolites that play a role in ARDS [2]. Our study has revealed that controlling the uptake of Phenylalanine might be a novel strategy for treating ARDS. This would also open new avenues in the control of pathological inflammatory responses. Sufficient (high-dose) protein was suggested to be provided in critically ill patients [33], nevertheless, eight essential amino acids including Phenylalanine were indispensable 
in our commonly used parenteral and enteral nutrition. To reduce the uptake of Phenylalanine, the Phenylalaninerestricted diet designed for phenylketonuria patients might be a good choice. It is a feasible and easy approach that can be implemented in ARDS patients.

The present study still has some limitations. The small samples size of our ARDS patients was the main one. However, the matched age and gender ratio as well as the strict screening standards by multivariate and univariate analysis minimized the artificial mistakes. Secondly, the commonly used drugs in critically ill individuals could also be detected in our analysis. It was difficult to interpret the role of these metabolites, therefore, we didn't include them in our list of differential metabolites and focused only on the endogenous compounds. Thirdly, the nutritional status of ARDS and control subjects was not controlled for, which could affected the results of metabolomics. Lastly, the untargeted screening by metabolomics can only obtain relative concentrations of metabolites, quantitative metabolomics should be used in the future studies to confirm the absolute concentration of Phenylalanine and its related metabolites in ARDS patients.

\section{Conclusions}

In conclusion, our study revealed that the perturbance of Phenylalanine metabolism was associated with the rate of mortality in ARDS. Phenylalanine increased in the ARDS patients compared to the healthy controls, and in the nonsurvivors compared to the survivors of ARDS. Moreover, our study is the first to demonstrate high levels of Phenylalanine were associated with the aggravated lung injury and increased mortality of ARDS mice.

\section{Supplementary information}

Supplementary information accompanies this paper at https://doi.org/10 1186/s12931-020-01364-6.

\section{Additional file 1 \\ Additional file 2. \\ Additional file 3.}

\section{Abbreviations}

ARDS: Acute respiratory distress syndrome; ICU: Intensive care unit; BALF: Bronchoalveolar lavage fluid; QC: Quality control; UHPLC-MS/MS: Ultra High Pressure Liquid Chromatography -Mass Spectrum/ Mass Spectrum; KEGG: Kyoto Encycloa of Genes and Genomes; HMDB: Human Metabolome Database; APACHE: Acute physiologic and chronic health evaluation; CRP: Creactive protein; PCT: Procalcitonin; PCA: Principal component analysis; PLSDA: Partial least square-discriminant Analysis; VIP: Variable importance in projection; HE: Hematoxylin-eosin staining; PHA: Phenylalanine 4-hydroxylase; BH4: 5,6,7,8-tetrahydrobiopterin

\section{Acknowledgements}

We would like to thank all the patients and guardians of those patients for their participation in the study We would also like to acknowledge the professors and colleagues from Shanghai Jiaotong University including: Yingjie-Xu (Department of Biochemistry and Molecular Cell Biology), Jingjing Zhang (Ruijin hospital), Jiahui Wang (Department of Biochemistry and
Molecular Cell Biology), Jiahui Zhang (Department of Biochemistry and Molecular Cell Biology), JiaXia (Xinhua hospital), Youhuan Zhong (Department of Biochemistry and Molecular Cell Biology),

\section{Authors' contributions}

JX enrolled the patients in the project, analyzed the data of metabolomics, performed the mice experiments and drafted the manuscript; TT-P analyzed the data, edited the manuscript. XL-Q assisted in parts of the experiments; and RM-T helped enrolled the patients in the project; ZJ-L and HP-Q and XL$W$ helped in the draft of the article; YZ helped analyze the statistics; $H C$ and $\mathrm{YH}-\mathrm{W}$ helped in the targeted metabolomics assay; ZJJ participated in the mice experiments; JW performed HE staining; $J L-L$ designed the experiments and revised the article. All authors read and approved the final manuscript.

\section{Funding}

This study was supported by National Natural Science Foundation of China $(8177010121,8197010190)$ awarded to JL-L, Key national R \& D projects during the 13th five year plan (2016YFC1304303) awarded to JL-L, National Natural Science Foundation of China (8180080170) awarded to TT-P, and (81772040) awarded to HP-Q. Shanghai Sailing Program

(18YF1413800) awarded to TT-P.

Availability of data and materials

The datasets used and/or analyzed during the current study are available from the corresponding author on reasonable request.

\section{Ethics approval and consent to participate}

The human study was approved by the Ruijin Hospital Ethics Committee Shanghai Jiao Tong University School of Medicine. This trial is registered with the Chinese Clinical Trial Registry under number ChiCTR1800015930. The animal study was approved by the University Committee for Laboratory Animals and was performed in accordance with the guidelines of the Shanghai Institutes for Biological Sciences Council on Animal Care.

\section{Consent for publication}

Not applicable.

\section{Competing interests}

The authors declare that they have no competing interests.

\section{Author details}

'Department of Critical Care Medicine, Ruijin Hospital, Shanghai Jiao Tong University School of Medicine, Shanghai, China. ${ }^{2}$ Department of Pulmonary and Critical Care Medicine, Center of Respiratory Medicine, China-Japan Friendship Hospital, Beijing, China. ${ }^{3}$ Department of Pulmonary Medicine, Ruijin Hospital, Shanghai Jiao Tong University School of Medicine, Shanghai, China. ${ }^{4}$ Department of Emergency Medicine, Ruijin Hospital, Shanghai Jiao Tong University School of Medicine, Shanghai, China. ${ }^{5}$ Department of Gynecology and Obstetrics, Ruijin Hospital, Shanghai Jiao Tong University School of Medicine, Shanghai, China. ${ }^{6}$ Department of Biochemistry and Molecular Cell Biology, Shanghai Jiao Tong University School of Medicine, Shanghai, China.

Received: 18 November 2019 Accepted: 16 April 2020

Published online: 30 April 2020

\section{References}

1. Bellani G, Laffey JG, Pham T, Fan E, Brochard L, Esteban A, Gattinoni L, van Haren F, Larsson A, McAuley DF, et al. Epidemiology, patterns of care, and mortality for patients with acute respiratory distress syndrome in intensive care units in 50 countries. JAMA. 2016;315:788-800.

2. Wishart DS. Metabolomics for investigating physiological and pathophysiological processes. Physiol Rev. 2019;99:1819-75.

3. Lin S, Yue X, Wu H, Han TL, Zhu J, Wang C, Lei M, Zhang M, Liu Q, Xu F. Explore potential plasma biomarkers of acute respiratory distress syndrome (ARDS) using GC-MS metabolomics analysis. Clin Biochem. 2019;66:49-56.

4. Viswan A, Ghosh P, Gupta D, Azim A, Sinha N. Distinct metabolic Endotype mirroring acute respiratory distress syndrome (ARDS) subphenotype and its heterogeneous biology. Sci Rep. 2019;9:2108.

5. Evans CR, Karnovsky A, Kovach MA, Standiford TJ, Burant CF, Stringer KA. Untargeted LC-MS metabolomics of bronchoalveolar lavage fluid 
differentiates acute respiratory distress syndrome from health. J Proteome Res. 2014;13:640-9.

6. Rogers AJ, Contrepois K, Wu M, Zheng M, Peltz G, Ware LB, Matthay MA. Profiling of ARDS pulmonary edema fluid identifies a metabolically distinct subset. Am J Physiol Lung Cell Mol Physiol. 2017;312:L703-9.

7. Liu PS, Wang H, Li X, Chao T, Teav T, Christen S, Di Conza G, Cheng WC, Chou CH, Vavakova M, et al. Alpha-ketoglutarate orchestrates macrophage activation through metabolic and epigenetic reprogramming. Nat Immunol. 2017;18:985-94

8. Murphy MP, O'Neill LAJ. Krebs cycle reimagined: the emerging roles of succinate and Itaconate as signal transducers. Cell. 2018;174:780-4.

9. Tojo K, Tamada N, Nagamine Y, Yazawa T, Ota S, Goto T. Enhancement of glycolysis by inhibition of oxygen-sensing prolyl hydroxylases protects alveolar epithelial cells from acute lung injury. FASEB J. 2018:fj201700888R. https://doi.org/10.1096/fj.201700888R.

10. Force ADT, Ranieri VM, Rubenfeld GD, Thompson BT, Ferguson ND, Caldwell E, Fan E, Camporota L, Slutsky AS. Acute respiratory distress syndrome: the Berlin definition. JAMA. 2012;307:2526-33.

11. Yi WJ, Han YS, Wei LL, Shi LY, Huang H, Jiang TT, Li ZB, Chen J, Hu YT, Tu HH, Li JC. I-Histidine, arachidonic acid, biliverdin, and I-cysteine-glutathione disulfide as potential biomarkers for cured pulmonary tuberculosis. Biomed Pharmacother. 2019;116:108980.

12. Sikalidis AK. Amino acids and immune response: a role for cysteine, glutamine, phenylalanine, tryptophan and arginine in T-cell function and cancer? Pathol Oncol Res. 2015:21:9-17.

13. Levy HL, Sarkissian CN, Scriver CR. Phenylalanine ammonia lyase (PAL): from discovery to enzyme substitution therapy for phenylketonuria. Mol Genet Metab. 2018;124:223-9.

14. Ploder M, Neurauter G, Spittler A, Schroecksnadel K, Roth E, Fuchs D. Serum phenylalanine in patients post trauma and with sepsis correlate to neopterin concentrations. Amino Acids. 2008;35:303-7.

15. Murr C, Grammer TB, Meinitzer A, Kleber ME, Marz W, Fuchs D. Immune activation and inflammation in patients with cardiovascular disease are associated with higher phenylalanine to tyrosine ratios: the ludwigshafen risk and cardiovascular health study. J Amino Acids. 2014;2014:783730.

16. Neurauter G, Grahmann AV, Klieber M, Zeimet A, Ledochowski M, SpernerUnterweger B, Fuchs D. Serum phenylalanine concentrations in patients with ovarian carcinoma correlate with concentrations of immune activation markers and of isoprostane-8. Cancer Lett. 2008;272:141-7.

17. Huang SS, Lin JY, Chen WS, Liu MH, Cheng CW, Cheng ML, Wang CH. Phenylalanine- and leucine-defined metabolic types identify high mortality risk in patients with severe infection. Int J Infect Dis. 2019;85:143-9.

18. Neurauter G, Schrocksnadel K, Scholl-Burgi S, Sperner-Unterweger B, Schubert C, Ledochowski M, Fuchs D. Chronic immune stimulation correlates with reduced phenylalanine turnover. Curr Drug Metab. 2008;9: 622-7.

19. Strasser B, Sperner-Unterweger B, Fuchs D, Gostner JM. Mechanisms of inflammation-associated depression: immune influences on tryptophan and phenylalanine metabolisms. Curr Top Behav Neurosci. 2017;31:95-115.

20. Dong Y, Bai Y, Liu G, Wang Z, Cao J, Chen Y, Yang H. The immunologic and antioxidant effects of $\mathrm{L}$-phenylalanine on the uterine implantation of mice embryos during early pregnancy. Histol Histopathol. 2014;29:1335-42.

21. Jiang M, Gong QY, Lai SS, Cheng ZX, Chen ZG, Zheng J, Peng B. Phenylalanine enhances innate immune response to clear ceftazidimeresistant Vibrio alginolyticus in Danio rerio. Fish Shellfish Immunol. 2019;84: 912-9.

22. Han S, Mallampalli RK. The acute respiratory distress syndrome: from mechanism to translation. J Immunol. 2015;194:855-60.

23. Poesen R, Claes K, Evenepoel P, de Loor H, Augustijns P, Kuypers D, Meijers B. Microbiota-derived Phenylacetylglutamine associates with overall mortality and cardiovascular disease in patients with CKD. J Am Soc Nephrol. 2016;27:3479-87.

24. Dixon B. The role of microvascular thrombosis in sepsis. Anaesth Intensive Care. 2004;32:619-29.

25. Evans CE, Zhao YY. Impact of thrombosis on pulmonary endothelial injury and repair following sepsis. Am J Physiol Lung Cell Mol Physiol. 2017;312: L441-51.

26. Nagendran M, McAuley DF, Kruger PS, Papazian L, Truwit JD, Laffey JG Thompson BT, Clarke M, Gordon AC. Statin therapy for acute respiratory distress syndrome: an individual patient data meta-analysis of randomised clinical trials. Intensive Care Med. 2017;43:663-71.
27. Polverino E, Rosales-Mayor E, Dale GE, Dembowsky K, Torres A. The role of neutrophil Elastase inhibitors in lung diseases. Chest. 2017;152:249-62.

28. Aschner Y, Zemans RL, Yamashita CM, Downey GP. Matrix metalloproteinases and protein tyrosine kinases: potential novel targets in acute lung injury and ARDS. Chest. 2014;146:1081-91.

29. Shaw TD, McAuley DF, O'Kane CM. Emerging drugs for treating the acute respiratory distress syndrome. Expert Opin Emerg Drugs. 2019;24:29-41.

30. Malaviya R, Laskin JD, Laskin DL. Anti-TNFa therapy in inflammatory lung diseases. Pharmacol Ther. 2017;180:90-8.

31. Matthay MA, Zemans RL, Zimmerman GA, Arabi YM, Beitler JR, Mercat A, Herridge M, Randolph AG, Calfee CS. Acute respiratory distress syndrome. Nat Rev Dis Primers. 2019;5:18.

32. Zhu D, Zhang Y, Li S, Gan L, Feng H, Nie W. Enteral omega-3 fatty acid supplementation in adult patients with acute respiratory distress syndrome: a systematic review of randomized controlled trials with meta-analysis and trial sequential analysis. Intensive Care Med. 2014;40:504-12.

33. McClave SA, Taylor BE, Martindale RG, Warren MM, Johnson DR, Braunschweig C, McCarthy MS, Davanos E, Rice TW, Cresci GA, et al. Guidelines for the provision and assessment of nutrition support therapy in the adult critically ill patient: Society of Critical Care Medicine (SCCM) and American Society for Parenteral and Enteral Nutrition (A.S.P.E.N.). JPEN J Parenter Enteral Nutr. 2016:40:159-211.

\section{Publisher's Note}

Springer Nature remains neutral with regard to jurisdictional claims in published maps and institutional affiliations.

\section{Ready to submit your research? Choose BMC and benefit from:}

- fast, convenient online submission

- thorough peer review by experienced researchers in your field

- rapid publication on acceptance

- support for research data, including large and complex data types

- gold Open Access which fosters wider collaboration and increased citations

- maximum visibility for your research: over $100 \mathrm{M}$ website views per year

At BMC, research is always in progress.

Learn more biomedcentral.com/submissions 